\title{
АНАЛИЗ ЗАБОЛЕВАЕМОСТИ ЛИМФОМОЙ ХОДЖКИНА И НЕХОДЖКИНСКИМИ ЛИМФОМАМИ В РЕСПУБЛИКЕ БЕЛАРУСЬ
}

\author{
(C) Чешик A.A.
}

\begin{abstract}
Государственный регистр лиц, подвергшихся воздействию радиации вследствие катастрофы на Чернобыльской АЭС Республиканского научно-практического центра радиационной медицины и экологии человека, Гомель, Республика Беларусь
\end{abstract}

E-mail: acheshik@tut.by

\begin{abstract}
Целью работы явилась оценка заболеваемости лимфомой Ходжкина (МКБ10: С81.0 - С81.9) и неходжкинскими лимфомами (МКБ10: С82.0 - С85.9, С96) в Республике Беларусь в 1991-2014 гг. В работе были проанализированы данные Белорусского республиканского канцер-регистра за период с 1991 г. по 2014 г. Рассчитаны грубые интенсивные (CR), повозрастные (AsR) и стандартизованные по возрасту (ASR) показатели заболеваемости (на 100000 населения). Анализ динамик показателей проводился путем сравнения темпов среднегодового прироста (АРC). За период с 1991 г. по 2014 год в Республике Беларусь зарегистрировано 6675 случаев лимфомы Ходжкина и 12404 случая неходжкинской лимфомы. Анализ CR и ASR показателей показал, что заболеваемость лимфомами у мужчин выше, чем у женщин, а у городских жителей выше, чем у сельских. Исключением стала ASR заболеваемость лимфомой Ходжкина, где она незначительно выше была у женщин.
\end{abstract}

Ключевые слова: лимфома Ходжкина, неходжкинские лимфомы, заболеваемость, стандартизованный показатель, грубый интенсивный показатель.

\section{HODGKIN LYMPHOMA AND NON-HODGKIN LYMPHOMAS INCIDENCE RATES IN THE REPUBLIC OF BELARUS Cheshik A.A.}

State Register of Persons Exposed to Radiation as a Result of the Chernobyl Disaster

of Republican Research Center for Radiation Medicine and Human Ecology, Gomel, Republic of Belarus

Hodgkin lymphoma and Non-Hodgkin lymphomas are those of the most expected malignant neoplasms, which could be associated with radiation impact. There have been no epidemiological studies, which would describe lymphoma rates in Belarus for the long time after the Chernobyl disaster. Thus the aim of this study was to estimate lymphomas rates in Belarus within 1991-2014. Data of Belorussian Cancer Registry were analyzed by calculation of crude rates (CR), age-specific rates (AsR) and age standardized rates (ASR). Trend analysis was realized by investigation of average annual percentage change (APC). There were found 6,675 cases of Hodgkin lymphoma (C81.0 - C81.9) and 12,404 of Non-Hodgkin lymphomas (C82.0 C85.9, C96) in Belarus within 1991-2014. CR and ASR of lymphomas are higher in males than females, but the return ASR of Hodgkin lymphoma is higher in females than males. CR and ASR are higher for urban than rural residents. Trend analysis showed the growth of both crude and standardized incidence rates of Non-Hodgkin lymphomas (APC is about 3.4\%), but the return APS of Hodgkin lymphoma made up $(-0.2 \%)$.

Keywords: Hodgkin lymphoma and Non-Hodgkin lymphomas, standardized incidence rates, crude incidence rates.

По данным Международного агентства по изучению рака (МАИР) [3] заболеваемость лимфомой Ходжкина (лимфогранулематоз) (далее ЛГМ) в мировой популяции мужчин в 2012 году составила $1,1 \% 0000$, у женщин $-0,7 \% 0000$. В то же время отмечено, что показатель заболеваемости у мужчин в развивающихся странах $(0,8 \% 000)$ почти в три раза ниже, чем у мужского населения развитых стран $(2,3 \% 0000)$. Среди женского населения различия в уровнях заболеваемости в развитых $(1,9 \%$ \% ходятся примерно на том же уровне, что и у мужчин.

Заболеваемость неходжкинскими лимфомами (далее - НХЛ) в мировой популяции мужчин в 2012 году составила $6,0 \%$, у женщин $-4,1 \%$, 0000 . Показатель заболеваемости у мужчин в развивающихся странах $(4,3 \% 0000)$ в 2,5 раза ниже, чем у мужского населения развитых стран $(10,3 \% 000)$. Среди женского населения различия в уровнях заболеваемости в развитых $(7,1 \% 000)$ и развивающихся $(2,8 \%$ о000) странах также находятся примерно на том же уровне, что и у мужчин.

Проведенный нами анализ литературных источников показал небольшое количество публикаций, характеризующих тенденции заболеваемости ЛГМ и НХЛ в Республике Беларусь в послеаварийный период. Знание особенностей заболеваемости этими заболеваниями в Беларуси важно для решения задач по оценке радиационно обусловленных рисков развития этих заболеваний у пострадавшего населения. В связи с этим целью данной работы было провести анализ заболеваемости ЛГМ и НХЛ в Республике Беларусь за период с 1991 г. по 2014 г. 


\section{МАТЕРИАЛЫ И МЕТОДЫ ИССЛЕДОВАНИЯ}

В работе были проанализированы данные Белорусского республиканского канцер-регистра за период с 1991 г. по 2014 г. В данной работе устанавливались особенности заболеваемости лимфомой Ходжкина (МКБ10: С81.0 - С81.9) и неходжкинскими лимфомами (МКБ10: С82.0 - С85.9, С96). В работе была использована международная классификация болезней 10 пересмотра. Были рассчитаны грубые интенсивные (CR), повозрастные (AsR) и стандартизованные по возрасту (ASR, World стандарт) показатели заболеваемости (на 100000 населения). Статистическая обработка материала проводилась в пакете Excel c использованием сравнения 95\% доверительных интервалов (95\%ДИ), рассчитанных с использованием Z-критерия и стандартной ошибки показателя $(\mathrm{SE})[1,2]$. Анализ динамик показателей заболеваемости проводился путем сравнения темпов среднегодового прироста (АРС) с использованием пакета Joinpoint Regression Program 3.4.3.

\section{РЕЗУЛЬТАТЫ ИССЛЕДОВАНИЯ И ИХ ОБСУЖДЕНИЕ}

В структуре заболеваемости населения Беларуси злокачественными новообразованиями ЛГМ на протяжении 1991-2014 годов составляла порядка $0,7-1,0 \%$ как у мужчин, так и у женщин, а НХЛ $1,4 \%$ у мужчин и женщин соответственно.

За период с 1991 г. по 2014 г. в Республике Беларусь зарегистрировано 6675 случаев ЛГМ и 12404 случая НХЛ. Соотношение числа заболевших ЛГМ мужчин и женщин за это время составило $0,92 / 1$ и НХЛ 1,02/1 соответственно.

На рисунке 1 приведены повозрастные показатели заболеваемости ЛГМ в Республике Беларусь за период с 1991 г. по 2014 г. Как видно из рисунка, случаи заболеваемости ЛГМ отмечаются уже в детском возрасте как у лиц женского, так и мужского пола. С течением возраста отмечается увеличение заболеваемости с достижением максимума в возрастной группе 20-24 лет у мужчин $(4,4 \pm 0,44 \%$ и $(6,2 \pm 0,53 \%$ о0о0). После 30 лет наблюдается спад заболеваемости с последующим ростом. После 45 лет риск заболеть ЛГМ увеличивается, достигая второго максимума заболеваемости в возрасте $70-74$ года у мужчин $(2,9 \pm 0,1 \%$ о женщин $(2,8 \pm 0,09 \%$ \%000). После 75 лет заболеваемость ЛГМ начинает снижаться.

Показатели заболеваемости ЛГМ незначительно выше у мужчин, чем у женщин, статистически значимо в возрастных группах 0-9, 15-34, $45-74$ и в $80-84$ лет $(\mathrm{p}<0,05)$.
На рисунке 2 приведены повозрастные показатели заболеваемости НХЛ в Республике Беларусь за период с 1991 г. по 2014 г.

Анализ повозрастных показателей заболеваемости НХЛ показал, что небольшой пик заболеваемости отмечается в возрасте 5-9 лет, после чего риск заболеть НХЛ увеличивается с возрастом, достигая максимума в возрастной группе $75-79$ лет у мужчин $(25,1 \pm 2,33 \%$ \%о00) и у женщин $(16,7 \pm 1,21 \%$ о000). После 80 лет заболеваемость НХЛ начинает снижаться.

Показатели заболеваемости НХЛ статистически значимо выше у мужчин, чем у женщин, во всех возрастных группах $(\mathrm{p}<0,05)$, за исключением возрастной группы 20-24 года $(\mathrm{p}=0,34)$.

За период с 1991 по 2014 годы в Республике Беларусь зарегистрировано 6675 случаев ЛГМ. Соотношение числа заболевших мужчин и женщин за это время составило 0,92/1.

В течение всего периода с 1991 г. по 2014 г. не наблюдалось значительных изменений количества ежегодно выявляемых случаев лимфомы Ходжкина на территории РБ (среднегодовой темп прироста составил $-0,2(-0,6-0,26)(\mathrm{p}>0,05))$.

В отличие от ЛГМ, у НХЛ в течение всего периода наблюдался рост числа ежегодно заболевших НХЛ на территории РБ. Так, число ежегодно регистрируемых новых случаев заболевания в 1991 году составило 339 (192 у мужчин и 147 у женщин), а в 2014 - 721 случай (345 у мужчин и 376 у женщин) (среднегодовой темп прироста составил $3,2(2,74-3,6) \%(p<0,05))$.

В таблице 1 приведены динамики грубых интенсивных и стандартизованных показателей заболеваемости ЛГМ и НХЛ мужчин и женщин, городских и сельских жителей.

Динамика грубого интенсивного показателя заболеваемости ЛГМ имеет статистически значимый положительный среднегодовой темп прироста у женщин $0,7 \%$, и отрицательный у мужчин $-0,2 \%$. Так, грубый интенсивный показатель заболеваемости у женщин незначительно вырос с 2,6士 $0,43^{0} \%_{0000}$ в 1991 г. до $2,8 \pm 0,46^{0} \% 000$ в 2014 г. (АРC $=$ $0,7(0,14-1,24) \%(p<0,05)$. При этом у городских жителей не наблюдалось значительного изменения заболеваемости ЛГМ (АРC $=0,4 \quad(-0,31-1,08) \%$ $(\mathrm{p}>0,05))$, у жителей села отмечался слабый рост заболеваемости с $2,3 \pm 0,69 \%$ \% в 2014 г. $(\mathrm{APC}=1,4 \quad(0,14-2,63) \%(\mathrm{p}<0,05))$. У мужчин не отмечалось изменений показателя заболеваемости ЛГМ (АРС $=-0,2 \quad(-0,78-0,35) \%$ $(\mathrm{p}>0,05))$, заболеваемость колебалась в пределах $2,6 \pm 0,48-3,6 \pm 0,54 \%$ \%000 за весь период наблюдения. Данная тенденция прослеживалась как у городских жителей $\left(2,5 \pm 0,54^{0} \% 000-3,5 \pm 0,65^{0} \% 000 \quad\right.$ (АРC $=$ $0(-0,7-0,68) \% \quad(p>0,05)$, так и сельских жителей $(2,8 \pm 1,01 \%$ \% $00003,7 \pm 0,95 \%$ \%000 (APC $=-0,9 \quad(-1,82-$ $0,05) \%(\mathrm{p}>0,05))$. 


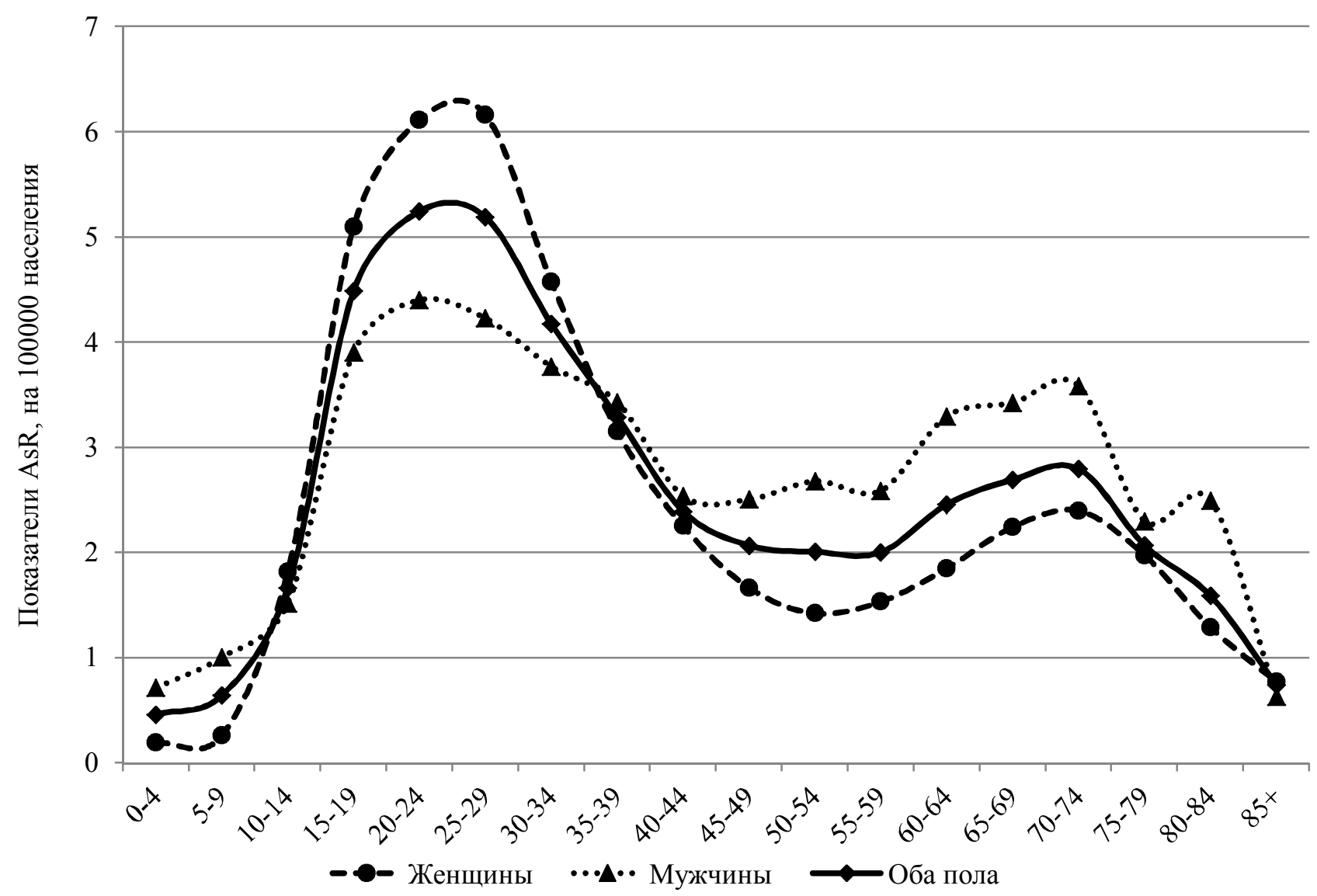

Рис. 1. Повозрастные показатели заболеваемости (AsR 95\%ДИ) ЛГМ в Республике Беларусь за период с 1991 г. по 2014 г.

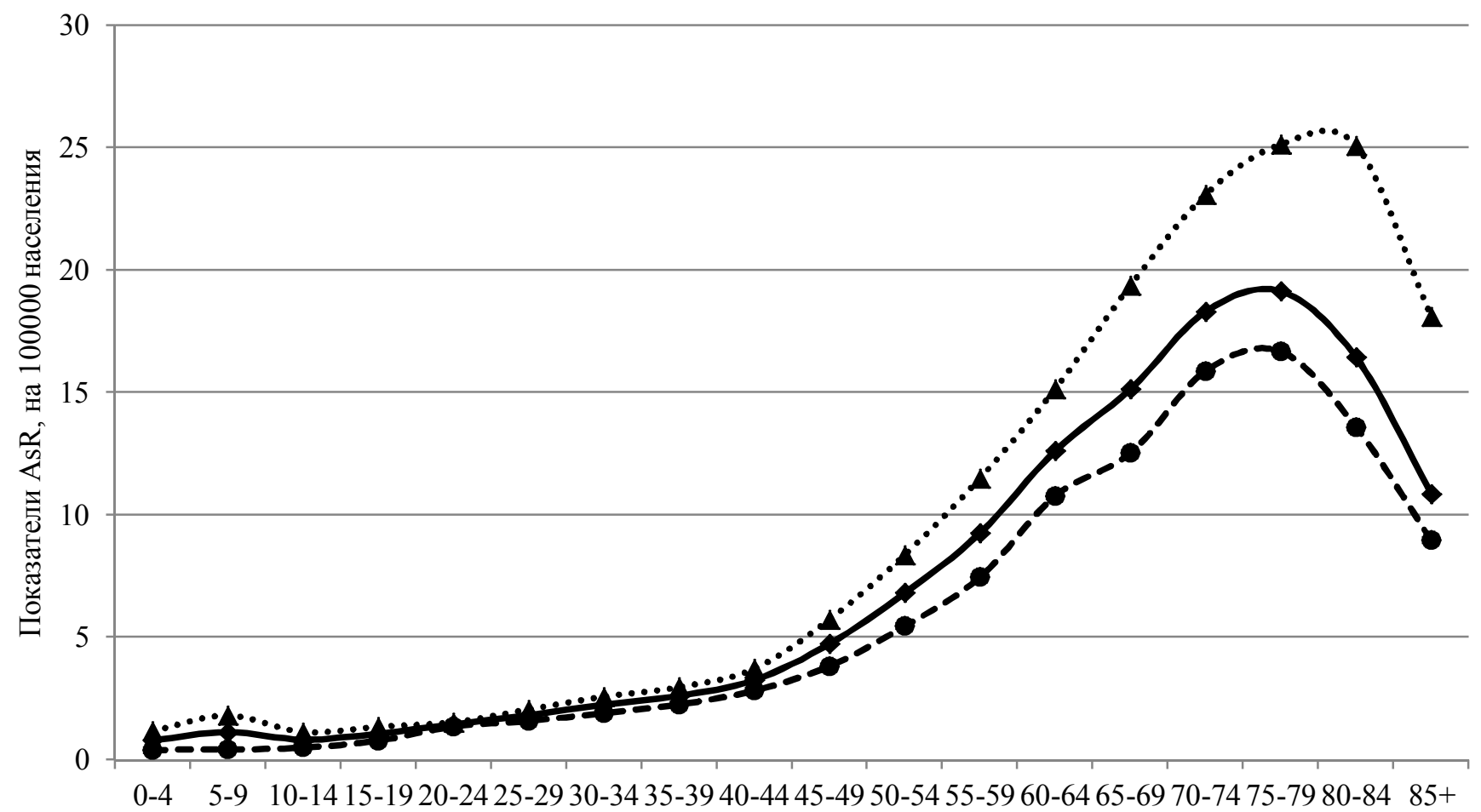

- - Женщины ….. Мужчины — Оба пола

Рис. 2. Повозрастные показатели заболеваемости (AsR 95\%ДИ) НХЛ в Республике Беларусь за период с 1991 г. по 2014 г. 
Таблица 1

Динамики грубых интенсивных и стандартизованных показателей заболеваемости ЛГМ и НХЛ мужчин и женщин, городских и сельских жителей, темпы среднегодового прироста

\begin{tabular}{|c|c|c|c|c|c|c|c|c|c|}
\hline \multirow{3}{*}{ 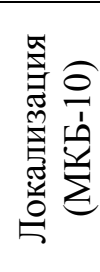 } & \multirow{3}{*}{$\begin{array}{c}\text { Пол/ } \\
\text { место } \\
\text { жительства }\end{array}$} & \multicolumn{4}{|c|}{$\begin{array}{c}\text { Грубый интенсивный показатель } \\
\text { заболеваемости } \\
\text { на } 100000 \text { населения } \pm \mathrm{Z} \times \mathrm{Se} \\
\end{array}$} & \multicolumn{4}{|c|}{$\begin{array}{c}\text { Стандартизованный показатель } \\
\text { заболеваемости } \\
\text { на } 100000 \text { населения } \pm \mathrm{Z} \times \mathrm{Se} \\
\end{array}$} \\
\hline & & \multicolumn{4}{|c|}{ годы } & \multicolumn{4}{|c|}{ годы } \\
\hline & & 1991 & 2001 & 2014 & $\begin{array}{c}\text { АРС } \\
(95 \% Д И)\end{array}$ & 1991 & 2001 & 2014 & $\begin{array}{c}\text { АРС } \\
(95 \% Д И)\end{array}$ \\
\hline \multirow{6}{*}{ 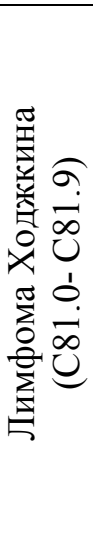 } & $\begin{array}{c}\text { Женщины/ } \\
\text { все }\end{array}$ & $\begin{array}{c}2,6 \\
\pm 0,43 \\
\end{array}$ & $\begin{array}{c}2,7 \\
\pm 0,45 \\
\end{array}$ & $\begin{array}{c}2,8 \\
\pm 0,46 \\
\end{array}$ & $\begin{array}{c}0,7 \\
(0,14-1,24)^{*} \\
\end{array}$ & $\begin{array}{r}2,6 \\
\pm 0,49 \\
\end{array}$ & $\begin{array}{c}2,7 \\
\pm 0,61 \\
\end{array}$ & $\begin{array}{c}2,9 \\
\pm 0,96 \\
\end{array}$ & $\begin{array}{c}0,5 \\
(-0,03-0,97)\end{array}$ \\
\hline & $\begin{array}{c}\text { Женщины/ } \\
\text { город }\end{array}$ & $\begin{array}{c}2,8 \\
\pm 0,55 \\
\end{array}$ & $\begin{array}{c}3,1 \\
\pm 0,57\end{array}$ & $\begin{array}{c}2,8 \\
\pm 0,52 \\
\end{array}$ & $\begin{array}{c}0,4 \\
(-0,31-1,08)\end{array}$ & $\begin{array}{c}2,6 \\
\pm 0,42 \\
\end{array}$ & $\begin{array}{c}2,9 \\
\pm 1,23 \\
\end{array}$ & $\begin{array}{c}2,7 \\
\pm 1,35 \\
\end{array}$ & $\begin{array}{c}0,2 \\
(-0,51-0,81)\end{array}$ \\
\hline & $\begin{array}{l}\text { Женщины/ } \\
\text { село }\end{array}$ & $\begin{array}{c}2,3 \\
\pm 0,69\end{array}$ & $\begin{array}{c}1,8 \\
\pm 0,67\end{array}$ & $\begin{array}{c}2,9 \\
\pm 0,98\end{array}$ & $\begin{array}{c}1,4 \\
(0,14-2,63)^{*}\end{array}$ & $\begin{array}{c}2,6 \\
\pm 0,89\end{array}$ & $\begin{array}{c}2 \\
\pm 0,7\end{array}$ & $\begin{array}{c}4,4 \\
\pm 1,69\end{array}$ & $\begin{array}{c}1,7 \\
(0,31-3,12)^{*}\end{array}$ \\
\hline & $\begin{array}{l}\text { Мужчины/ } \\
\text { все }\end{array}$ & $\begin{array}{c}3,6 \\
\pm 0,54\end{array}$ & $\begin{array}{c}3 \\
\pm 0,5\end{array}$ & $\begin{array}{c}2,6 \\
\pm 0,48\end{array}$ & $\begin{array}{c}-0,2 \\
(-0,78-0,35)\end{array}$ & $\begin{array}{c}3,5 \\
\pm 0,42\end{array}$ & $\begin{array}{c}2,7 \\
\pm 3,09\end{array}$ & $\begin{array}{c}2,3 \\
\pm 1,52\end{array}$ & $\begin{array}{c}-0,7 \\
(-1,24-0,06)^{*}\end{array}$ \\
\hline & $\begin{array}{l}\text { Мужчины/ } \\
\text { город }\end{array}$ & $\begin{array}{c}3,5 \\
\pm 0,65\end{array}$ & $\begin{array}{c}3,2 \\
\pm 0,61\end{array}$ & $\begin{array}{c}2,5 \\
\pm 0,54\end{array}$ & $\begin{array}{c}0 \\
(-0,7-0,68)\end{array}$ & $\begin{array}{c}3,6 \\
\pm 0,5\end{array}$ & $\begin{array}{c}2,9 \\
\pm 6,01\end{array}$ & $\begin{array}{c}2,2 \\
\pm 1,41\end{array}$ & $\begin{array}{c}-0,6 \\
(-1,33-0,12)\end{array}$ \\
\hline & $\begin{array}{l}\text { Мужчины/ } \\
\text { село }\end{array}$ & $\begin{array}{c}3,7 \\
\pm 0,95\end{array}$ & $\begin{array}{c}2,5 \\
\pm 0,84\end{array}$ & $\begin{array}{c}2,8 \\
\pm 1,01\end{array}$ & $\begin{array}{c}-0,9 \\
(-1,82-0,05)\end{array}$ & $\begin{array}{c}3,5 \\
\pm 0,77\end{array}$ & $\begin{array}{c}2,3 \\
\pm 2,62\end{array}$ & $\begin{array}{c}3 \\
\pm 3,37 \\
\end{array}$ & $\begin{array}{c}-0,9 \\
(-1,92-0,12)\end{array}$ \\
\hline \multirow{6}{*}{ 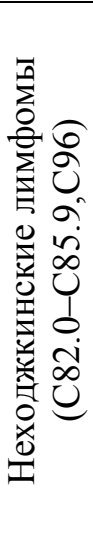 } & $\begin{array}{l}\text { Женщины/ } \\
\text { все }\end{array}$ & $\begin{array}{c}2,7 \\
\pm 0,44\end{array}$ & $\begin{array}{c}4,4 \\
\pm 0,57\end{array}$ & $\begin{array}{c}7,4 \\
\pm 0,75\end{array}$ & $\begin{array}{c}4,1 \\
(3,47-4,72)^{*}\end{array}$ & $\begin{array}{c}2 \\
\pm 0,25\end{array}$ & $\begin{array}{c}2,7 \\
\pm 0,27\end{array}$ & $\begin{array}{c}4,1 \\
\pm 0,55\end{array}$ & $\begin{array}{c}2,8 \\
(2,2-3,44)^{*}\end{array}$ \\
\hline & $\begin{array}{c}\text { Женщины/ } \\
\text { город }\end{array}$ & $\begin{array}{r}2,6 \\
\pm 0,52\end{array}$ & $\begin{array}{c}4,2 \\
\pm 0,66\end{array}$ & $\begin{array}{c}7,1 \\
\pm 0,84\end{array}$ & $\begin{array}{c}4,1 \\
(3,36-4,86)^{*}\end{array}$ & $\begin{array}{c}2,3 \\
\pm 0,31\end{array}$ & $\begin{array}{c}2,9 \\
\pm 0,32\end{array}$ & $\begin{array}{c}4,3 \\
\pm 0,82\end{array}$ & $\begin{array}{c}2,3 \\
(1,62-3,06)^{*}\end{array}$ \\
\hline & $\begin{array}{l}\text { Женщины/ } \\
\text { село }\end{array}$ & $\begin{array}{c}3 \\
\pm 0,8\end{array}$ & $\begin{array}{c}4,8 \\
\pm 1,09\end{array}$ & $\begin{array}{c}8,4 \\
\pm 1,68\end{array}$ & $\begin{array}{c}4,1 \\
(3,1-5,02)^{*}\end{array}$ & $\begin{array}{c}2,1 \\
\pm 0,56\end{array}$ & $\begin{array}{c}2,3 \\
\pm 0,52\end{array}$ & $\begin{array}{c}3,7 \\
\pm 0,72\end{array}$ & $\begin{array}{c}3 \\
(1,87-4,13)^{*}\end{array}$ \\
\hline & $\begin{array}{l}\text { Мужчины/ } \\
\text { все }\end{array}$ & $\begin{array}{c}4 \\
\pm 0,57\end{array}$ & $\begin{array}{c}5,2 \\
\pm 0,65\end{array}$ & $\begin{array}{c}7,8 \\
\pm 0,83\end{array}$ & $\begin{array}{c}3,1 \\
(2,61-3,57)^{*}\end{array}$ & $\begin{array}{c}3,8 \\
\pm 0,35\end{array}$ & $\begin{array}{c}4,5 \\
\pm 0,42\end{array}$ & $\begin{array}{c}6 \\
\pm 1,76\end{array}$ & $\begin{array}{c}2,2 \\
(1,75-2,7)^{*}\end{array}$ \\
\hline & $\begin{array}{c}\text { Мужчины/ } \\
\text { город }\end{array}$ & $\begin{array}{c}3,9 \\
\pm 0,68 \\
\end{array}$ & $\begin{array}{c}5,2 \\
\pm 0,78\end{array}$ & $\begin{array}{c}7,8 \\
\pm 0,94\end{array}$ & $\begin{array}{c}3,1 \\
(2,63-3,61)^{*}\end{array}$ & $\begin{array}{c}4,4 \\
\pm 0,45\end{array}$ & $\begin{array}{c}5,1 \\
\pm 0,53\end{array}$ & $\begin{array}{c}6,3 \\
\pm 2,4\end{array}$ & $\begin{array}{c}1,7 \\
(1,26-2,22)^{*}\end{array}$ \\
\hline & $\begin{array}{l}\text { Мужчины/ } \\
\text { село }\end{array}$ & $\begin{array}{c}4,3 \\
\pm 1,03\end{array}$ & $\begin{array}{c}5,1 \\
\pm 1,19\end{array}$ & $\begin{array}{c}8,1 \\
\pm 1,72\end{array}$ & $\begin{array}{c}3 \\
(2,13-3,96)^{*}\end{array}$ & $\begin{array}{c}3,3 \\
\pm 0,63\end{array}$ & $\begin{array}{c}3,7 \\
\pm 0,72\end{array}$ & $\begin{array}{c}5,5 \\
\pm 2,48\end{array}$ & $\begin{array}{c}2,6 \\
(1,54-3,7)^{*}\end{array}$ \\
\hline
\end{tabular}

Примечание: * $-\mathrm{p}<0,05$.

При этом заболеваемость ЛГМ на протяжении всего периода наблюдения у мужчин незначительно выше, чем у женщин, а у городских жителей была выше, чем у сельских. Статистически значимые различия $(\mathrm{p}<0,05)$ грубых интенсивных показателей заболеваемости ЛГМ у мужчин и женщин отмечаются в 1991 и 1999 гг. наблюдения. В то же время статистически значимые различия $(\mathrm{p}<0,05)$ грубых интенсивных показателей заболеваемости ЛГМ у мужчин, городских и сельских жителей отмечались в 2007, 2010 и 2012 гг. и у женщин в 1998, 2000-2004, 2007, 2009 и 2012 гг.

Как видно из таблицы динамики стандартизованных показателей заболеваемости, ЛГМ имеют отличия от динамик грубых интенсивных показателей. Статистически значимый спад показателей ASR отмечается для мужчин $-0,7 \%$, при этом для женщин села отмечается статистически значимый рост $(\mathrm{APC}=1,7(0,31-3,12)) \%$. Стандартизованные показатели заболеваемости у мужчин снизились с
$3,5 \pm 0,42 \%$ в 1991 г. до $2,3 \pm 1,52 \%$ в 2000 в 2014 г. $(\mathrm{APC}=-0,7(-1,24--0,06) \%(\mathrm{p}<0,05)$. При этом как у городских жителей не наблюдалось значительного изменения заболеваемости $3,6 \pm 0,5 \%$ о $2,2 \pm 1,41 \% \%_{0000}(\mathrm{APC}=-0,6(-1,33-0,12) \%(\mathrm{p}>0,05)$ так и у сельских $-3,5 \pm 0,77^{\circ} \% 0003 \pm 3,37 \%$ \%000 (АРС $=-0,9(-1,92-0,12) \%(\mathrm{p}>0,05)$. У женщин не отмечалось значительных изменений показателя заболеваемости ЛГМ (АРC $=0,5 \quad(-0,03-0,97) \%$ $(\mathrm{p}>0,05)$, она колебалась в пределах $2,3 \pm 0,54-$ $3,1 \pm 0,99 \%$ \%000. У городских жителей заболеваемость также колебалась в пределах 2,3 $\pm 1,13$ $3,3 \pm 1,99 \%$ \%000 $(\mathrm{APC}=0,2 \quad(-0,51-0,81) \%(\mathrm{p}>0,05)$. Однако у сельских жителей она выросла с $2,6 \pm 0,89 \%$ в 1900 д 1991 до $4,4 \pm 1,69 \%$ \%о0о в 2014 г. $(\mathrm{APC}=1,7(0,31-3,12) \%(\mathrm{p}<0,05)$. Следует отметить, что в отличие от грубых интенсивных показателей заболеваемости, стандартизованные по возрасту показатели незначительно выше у женщин, чем у мужчин. А показатель ASR, как и грубый интенсивный показатель городских, незначи- 
тельно выше, чем у сельских жителей, как у мужчин, так и у женщин.

Статистически значимых различий $(p<0,05)$ стандартизованных по возрасту показателей заболеваемости между мужчинами и женщинами отмечено не было. В то же время статистически значимые различия отмечались между заболеваемостью городских и сельских женщин $(p=0,018)$ только в 2000-2004 гг.

Динамика грубого интенсивного показателя заболеваемости НХЛ имеет положительные статистически значимые среднегодовые темпы прироста порядка $3 \%$ у мужчин и 4\% у женщин, жителей города и села. Так, показатель заболеваемости у мужчин вырос с 4,0 $\pm 0,57 \% 0000$ в 1991 г. до $7,8 \pm 0,83 \%$ в 2014 г. $($ АРC $=3,1 \quad(2,61-3,57) \%$ $(\mathrm{p}<0,05),($ у городских с $3,9 \pm 0,68 \% 0000$ в 1991 г. до $7,8 \pm 0,94 \%$ во00 в 2014 г. $($ АРC $=3,1 \quad(2,63-3,61) \%$ $(\mathrm{p}<0,05)$ и сельских с 4,3 $\pm 1,03 \%$ в 1991 г. до $8,1 \pm 1,72 \%$ в 2014 г. $($ APC $=3(2,13-3,96) \%$ $(\mathrm{p}<0,05))$. При этом у женщин показатель заболеваемости вырос с $2,7 \pm 0,44 \% 0000$ в 1991 г. до $7,4 \pm 0,75 \%$ в 2014 г. $($ APC $=4,1 \quad(3,47-4,72) \%$ $(\mathrm{p}<0,05)$, (у городских с 2,6 $\pm 0,52 \%$ в 1991 г. до $7,1 \pm 0,84 \%$ в 2014 г. $($ APC $=4,1 \quad(3,36-4,86) \%$ $(\mathrm{p}<0,05)$ и сельских с $3,0 \pm 0,8 \% 0000$ в 1991-г. до $8,4 \pm 1,68 \%$ в 2014 г. $($ АРС $=4,1 \quad(3,1-5,02) \%$ $(\mathrm{p}<0,05))$. При этом следует отметить, что грубый интенсивный показатель заболеваемости НХЛ на протяжении всего периода наблюдения выше у мужчин, чем у женщин, и оставался на одном уровне как у мужчин города и села, так и у женщин.

Статистически значимые различия $(\mathrm{p}<0,05)$ грубых интенсивных показателей заболеваемости лейкозами у мужчин и женщин отмечаются в 1991, 1993, 1995, 1996, 1997, 2002, 2004, 2005, 2006, 2011 гг. В то же время статистически значимые различия $(\mathrm{p}<0,05)$ грубых интенсивных показателей заболеваемости лейкозами у мужчин, городских и сельских жителей отмечались в 2003 и 2013 гг. и у женщин в 2013 году.

Как видно из таблицы динамики стандартизованных показателей НХЛ, все они, так же как и грубые интенсивные показатели, имеют статистически значимый рост. Так, стандартизованный показатель заболеваемости у мужчин вырос с $3,8 \pm 0,35 \%$ в 1991 г. до $6 \pm 1,76 \%$ в000 в 2014 г. $(\mathrm{APC}=2,2(1,75-2,7) \%(\mathrm{p}<0,05),($ у городских с $4,4 \pm 0,45 \%$ в 1991 г. до $6,3 \pm 2,4 \% 0000$ в 2014 г. $(\mathrm{APC}=1,7(1,26-2,22) \%(\mathrm{p}<0,05)$ и сельских с $3,3 \pm 0,63 \%$ в 1991 г. до $5,5 \pm 2,48 \%$ в 2014 г. $(\mathrm{APC}=2,6 \quad(1,54-3,7) \%(\mathrm{p}<0,05))$. При этом у женщин показатель заболеваемости вырос с $2 \pm 0,25 \%$ в 1991 г. до $4,1 \pm 0,55 \%$ в 2014 г. $(\mathrm{APC}=2,8(2,2-3,44) \%(\mathrm{p}<0,05),($ у городских с

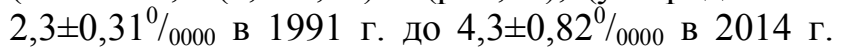

$(\mathrm{APC}=2,3 \quad(1,62-3,06) \%(\mathrm{p}<0,05)$ и сельских с $2,1 \pm 0,56 \%$ в 1991 г. до $3,7 \pm 0,72 \%$ в 2014 г. $(\mathrm{APC}=3(1,87-4,13) \%(\mathrm{p}<0,05))$. Следует отметить, что в отличие от грубых интенсивных показателей заболеваемости стандартизованные по возрасту показатели выше у городских, чем у сельских жителей, у мужчин и незначительно у женщин. При этом стандартизованный показатель заболеваемости, как и при сравнении грубых показателей, выше у мужчин, чем у женщин.

Статистически значимые различия $(\mathrm{p}<0,05)$ стандартизованных по возрасту показателей заболеваемости между мужчинами и женщинами отмечались во все годы наблюдения, а также между городскими и сельскими жителями, за исключением у женщин только в 2005-2009 гг. $(\mathrm{p}=0,101)$ и у мужчин в 2010-2014 гг. $(\mathrm{p}=0,165)$.

Таким образом, проведенный анализ заболеваемости за период с 1991 г. по 2014 г. позволяет сделать выводы о росте заболеваемости неходжкинскими лимфомами у населения Республики Беларусь в послеаварийный период со статистически значимым среднегодовым темпом прироста порядка $3,4 \%(\mathrm{p}<0,05)$. Тогда как в количестве ежегодно выявляемых случаев лимфомы Ходжкина на территории Республики Беларусь значительных изменений не наблюдалось (среднегодовой темп прироста составил $-0,2 \quad(-0,6-0,26)$ $(\mathrm{p}>0,05))$.

Анализ повозрастных показателей заболеваемости показал, что случаи заболеваемости ЛГМ отмечаются уже в детском возрасте как у лиц женского, так и мужского пола. С течением возраста отмечается увеличение заболеваемости с достижением максимума в возрастной группе $20-24$ лет у мужчин $(4,4 \pm 0,44 \% 0000)$ и в $25-29$ лет у женщин $(6,2 \pm 0,53 \%$ о000). После 30 лет наблюдается спад заболеваемости с последующим ростом. После 45 лет риск заболеть ЛГМ увеличивается, достигая второго максимума заболеваемости в возрасте $70-74$ года у мужчин $(2,9 \pm 0,1 \% 0000)$ и у женщин $\left(2,8 \pm 0,09^{0} \% 000\right)$. После 75 лет заболеваемость лимфомой Ходжкина начинает снижаться.

При анализе повозрастных показателей заболеваемости НХЛ было установлено, что небольшой пик заболеваемости отмечается в возрасте 59 лет, после чего риск заболеть НХЛ увеличивается с возрастом, достигая максимума в возрастной группе 75-79 лет у мужчин $(25,1 \pm 2,33 \%$ \%000) и у женщин $(16,7 \pm 1,21 \%$ \%000). После 80 лет заболеваемость НХЛ начинает снижаться.

Грубый интенсивный показатель заболеваемости ЛГМ на протяжении всего периода наблюдения у мужчин незначительно выше, чем у женщин, а у городских жителей был выше, чем у сельских. Грубый интенсивный показатель заболеваемости НХЛ выше был у мужчин, чем у жен- 
щин, и оставался на одном уровне как у мужчин города и села, так и у женщин соответственно.

Анализ стандартизованных по возрасту показателей ЛГМ показал, что заболеваемость незначительно выше у женщин, чем у мужчин, а у городских жителей незначительно выше, чем у сельских как у мужчин, так и у женщин. При этом стандартизованный показатель заболеваемости НХЛ выше у мужчин, чем у женщин. У мужчин и женщин жителей города выше, чем у сельских жителей.

\section{ЛИТЕРАТУРА}

1. Моисеев П.И., Веялкин И.В., Демидчик Ю.Е. Эпидемиология злокачественных новообразований: принципы и методы // Руководство по онкологии: учебник / под ред. О.Г. Суконко. - Минск, 2015. C. 51-82.

2. Breslow N.E., Day N.E. Statistical methods in cancer research. Volume II--The design and analysis of cohort studies. - Lyon: IARC, 1987. - Vol. 2. $404 \mathrm{p}$.

3. Globocan 2012: Estimated cancer incidence, mortality and prevalence worldwide in 2012 [Electronic resource] / Interntional Agency for Research of cancer. - France, 2012. - mode of access: http://globocan.iarc.fr. - date of access: 12.01.2016. 